Marquette University

e-Publications@Marquette

Clinical Lab Sciences Faculty Research and

Publications

Clinical Lab Sciences, Department of

10-15-2016

\title{
Update on Laboratory Diagnosis and Epidemiology of Trichomonas vaginalis: You Can Teach an "Old” Dog "New" Trichs
}

\author{
Erik Munson \\ Marquette University, erik.munson@marquette.edu \\ Maureen Napierala \\ Wheaton Franciscan Laboratory \\ Kimber L. Munson \\ Wheaton Franciscan Laboratory
}

Follow this and additional works at: https://epublications.marquette.edu/clinical_lab_fac

Part of the Laboratory and Basic Science Research Commons

\section{Recommended Citation}

Munson, Erik; Napierala, Maureen; and Munson, Kimber L., "Update on Laboratory Diagnosis and Epidemiology of Trichomonas vaginalis: You Can Teach an "Old" Dog "New" Trichs" (2016). Clinical Lab Sciences Faculty Research and Publications. 23.

https://epublications.marquette.edu/clinical_lab_fac/23 
Marquette University

e-Publications@Marquette

\section{Clinical Laboratory Sciences Faculty Research and Publications/College of Health Sciences}

This paper is NOT THE PUBLISHED VERSION; but the author's final, peer-reviewed manuscript. The published version may be accessed by following the link in th citation below.

Clinical Microbiology Newsletter, Vol. 38, No. 20 (October 15, 2016): 159-168. DOI. This article is (C) Elsevier and permission has been granted for this version to appear in e-Publications@Marquette. Elsevier does not grant permission for this article to be further copied/distributed or hosted elsewhere without the express permission from Elsevier.

\section{Update on Laboratory Diagnosis and Epidemiology of Trichomonas vaginalis: You Can Teach an "Old" Dog "New" Trichs}

\section{Erik Munson}

College of Health Sciences, Marquette University, Milwaukee, WI

Maureen Napierala

Wheaton Franciscan Laboratory, Milwaukee, WI

Kimber L. Munson

Wheaton Franciscan Laboratory, Milwaukee, WI

\section{Abstract}

Past viewpoints on Trichomonas vaginalis infection have characterized the associated clinical disease as a "nuisance" condition, with affected demographics largely being older African American females residing in urban centers. The advent of commercial molecular assays specific for T. vaginalishas offered a new outlook 
on trichomoniasis. Within high-prevalence sexually transmitted infection populations, parasite distribution is not localized to specific population centers, and T. vaginalis prevalence is elevated among both younger and older age groups. Adaptation of these molecular assays can additionally facilitate male screening and subsequent epidemiologic characterization. These findings, combined with associations between $T$. vaginalis infection and human immunodeficiency virus (HIV) acquisition/transmission and persistent human papillomavirus infection, support consideration of the expansion of $T$. vaginalis screening efforts in the realms of clinical practice and public health.

\section{Introduction}

The French microscopist, clinician, and researcher A. F. Donné discovered the protozoan $T$. vaginalis 180 years ago. Trichomoniasis, the clinical entity ascribed to the pathogen, has become the most prevalent non-viral sexually transmitted infection (STI) in the United States (Fig. 1). A surveillance study conducted by the U.S. Centers for Disease Control and Prevention (CDC) in 2008 estimated trichomoniasis prevalence in this country at 4 million cases, with approximately 1 million new cases of $T$. vaginalis infection being diagnosed annually. ${ }^{1}$ As will be posited and further described throughout this review, several factors impact the true characterization of trichomoniasis. As an introductory example, $T$. vaginalis prevalence rates are rather disparate on a worldwide basis. In contrast to reports of $>20 \%$ prevalence in some U.S., African, and indigenous Australian population centers, $, \underline{2}, \underline{4}, \underline{5}$ a number of European, Asian-Pacific, and non-indigenous Australian studies have documented rates lower than $5 \%, \underline{6}, \underline{7}, \underline{8}, \underline{9}, \underline{10}$

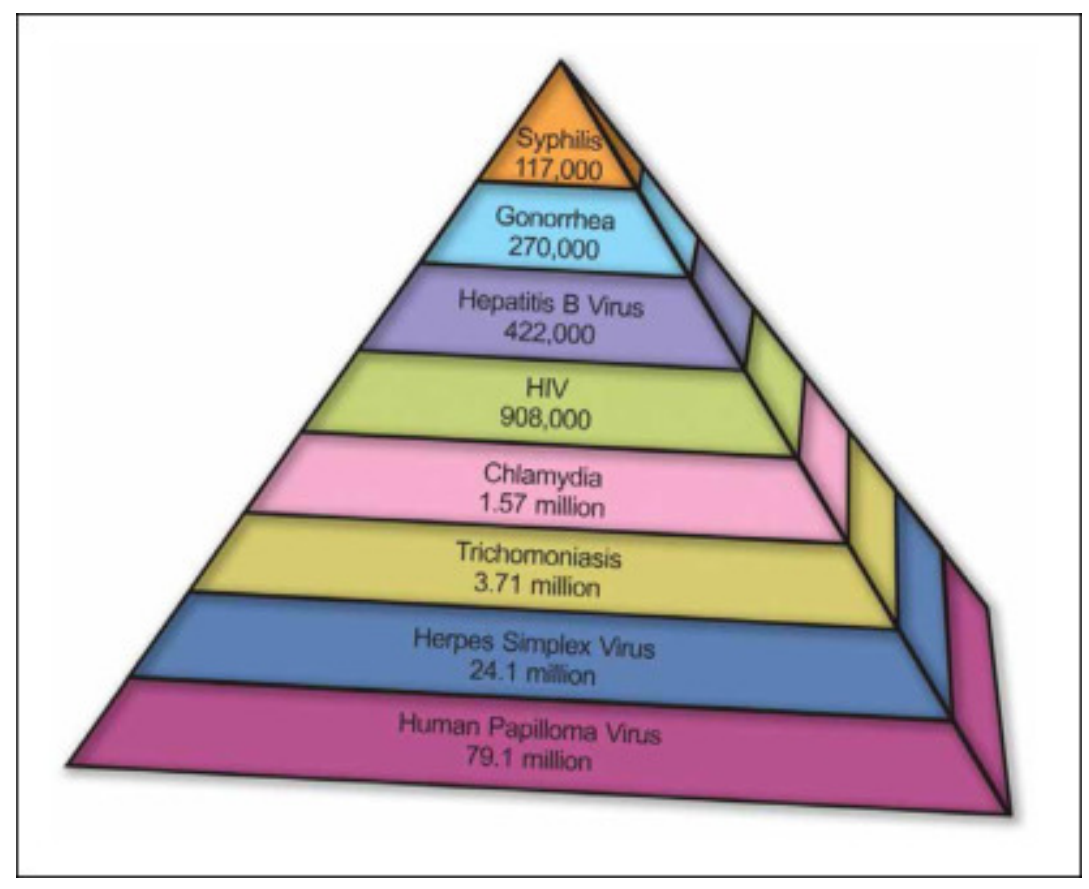

Figure 1. Estimated prevalence of STI in the United States (adapted from reference 75 with permission of the publisher). 
The advent of molecular-based laboratory detection of $T$. vaginalis has broadened our knowledge of trichomoniasis, allowing new perspectives on disease epidemiology and opportunities for prevention. In addition, advancements related to diagnostic algorithms have garnered much attention in the clinical research setting. A PubMed primary literature search (U.S. National Library of Medicine and the National Institutes of Health) conducted in February 2016 using the search parameter "Trichomonas

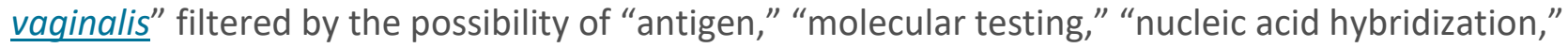
"PCR," "nucleic acid amplification testing," "LAMP" (loop-mediated isothermal amplification), or "transcription-mediated amplification" selected 629 primary citations from the years 1964 to 2016, with over $25 \%$ published since the beginning of 2012. This brief commentary, with focus largely on literature published within the past 3 years, discusses updates on T. vaginalisepidemiology and laboratory testing.

\section{Epidemiology}

\section{Females}

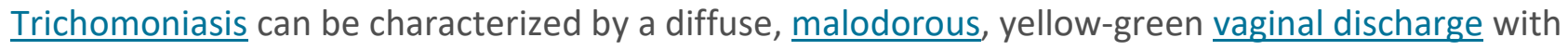
vulvar irritation. $\underline{11}$ While pruritis and dysuria may be present, a majority of $T$. vaginalis infections are actually thought to be asymptomatic. This has long been a source of consternation for clinicians and researchers in the context of proper laboratory diagnostic strategies and clinical management. Data published nearly 25 years ago stated that approximately one-third of patients with asymptomatic $T$. vaginalis infection become symptomatic within 6 months, $\underline{\underline{12}}$ perhaps necessitating additional encounters with health care. Mathematic modeling suggests that the average duration of $T$. vaginalis infection in women approaches 3 to 5 years. $\underline{13}$

Such data imply indolent $T$. vaginalis infection in a significant percentage of women, yet the organism has been associated with pregnancy complications, including preterm delivery, low-birthweight delivery, and delivery of offspring later diagnosed with intellectual disability or attention deficit-

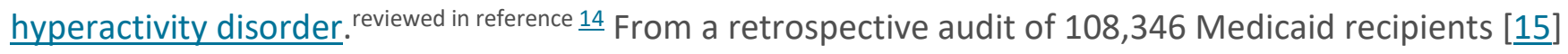
it was concluded that females diagnosed with trichomoniasis within the first 7 months of pregnancy were more likely to deliver at $\leq 33$ weeks of gestation (hazard ratio, 1.22 [95\% confidence interval, 1.02 to 1.46]), while those diagnosed within the first 8 months of pregnancy were more likely to deliver late pre-term (33 to 36 weeks; hazard ratio, 1.59 [95\% confidence interval, 1.18 to 2.14]). In single-site studies of South African and U.S. females, reviewed in reference $\underline{14}$ T. vaginalis associations with pelvic inflammatory disease in HIV-positive patients, acute endometritis, and tubal infertility have been reported. 
T. vaginalis has also achieved a status of importance in the public health community. The natural course of human papillomavirus (HPV) infection is largely self-limiting, with 60 to $80 \%$ of infections becoming undetectable in 8 to 10 months. $\underline{16}$ Prolonged or persistent HPV infections, particularly those involving high-risk HPV genotypes, contribute to ultimate development of cervical neoplasia. From an adolescent cohort, Shew et al. $\underline{16}$ demonstrated that median time to clearance of primary HPV infection was 172 days in patients without concomitant T. vaginalis infection. The median value increased to 436

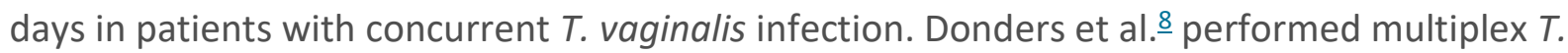

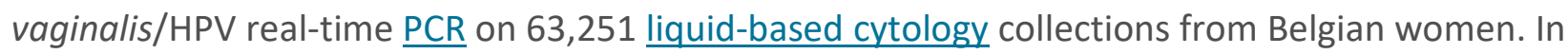
patients with detectable high-risk HPV DNA, the likelihood of having concomitant cytology findings of atypical squamous cells of uncertain significance or greater (ASC-US+) was higher when T. vaginalis was present than when T. vaginalis was absent (odds ratio, 1.98 [95\% confidence interval, 1.04 to 3.50]; $P=$ 0.049). Moreover, $17.8 \%$ of patients with detectable T. vaginalis DNA had cytology findings of ASC-US+, while only $8.7 \%$ of $T$. vaginalis-negative specimens had such findings (odds ratio, 2.3 [95\% confidence interval, 1.6 to 3.2]; $P<0.0001$ ). In an investigation of commercial sex workers in Kenya using RNA amplification techniques specific to both STI agents and high-risk HPV, Ting et al. $\underline{17}$ described higher rates of HPV positivity in women also positive for $T$. vaginalis.

The public health significance of $T$. vaginalis detection also extends to $\underline{\mathrm{HIV}}$. From a study of adolescents with behavioral risk factors for HIV infection, Mullins et al. $\underline{18}$ reported a higher $T$. vaginalis incidence rate in females who were HIV positive (1.3/100 person months) than in those who were not $(0.6 / 100$ person months; $P=0.002$ ). Several studies have characterized a role for $T$. vaginalis in HIV acquisition by females. One prospective study of 4,948 sexually active African females $\underline{19}$ reported that $T$. vaginalisinfected women were more likely to test positive for HIV at a subsequent health care encounter (adjusted hazard ratio, 2.05 [95\% confidence interval, 1.05 to 4.02]). Hughes et al. $\underline{20}$ prospectively studied 3,297 African HIV-serodiscordant couples and found that T. vaginalis infection of the female partner was an independent risk factor for increased probability of HIV acquisition per coital act (odds ratio, 2.57 [95\% confidence interval 1.42 to 4.65$]$ ).

In similar fashion, studies have elucidated a role for T. vaginalis in HIV transmission. Co-incubation of $T$. vaginalis with acutely infected peripheral blood mononuclear cells in vitro has been shown to increase the HIV replication rate. $\underline{21}$ A Louisiana cohort with documented infection by both HIV and $T$. vaginalis were prospectively observed following a course of metronidazole therapy. $\underline{22}$ Successful antitrichomonad therapy (determined by T. vaginalis culture) decreased the likelihood of HIV shedding at 3 months post-therapy compared to baseline shedding (relative risk, 0.34 [95\% confidence interval, 0.12 to 0.92]). This finding was not duplicated in HIV-positive women without antecedent $T$. vaginalis infection. Mathematical modeling of an HIV-infected population in North Carolina (with a 
presumed $22 \%$ T. vaginalis prevalence) predicted the occurrence of $0.076 \mathrm{HIV}$ transmission events per 100 HIV-positive females who test positive for T. vaginalis. $\underline{23}$ This datum contrasts with the rate of 0.062 HIV transmission events in HIV-positive women who are T. vaginalis negative. In the former scenario, $23 \%$ of HIV transmission events were attributable to T. vaginalisinfection.

Compared to commonly assessed STI agents, such as Chlamydia trachomatis and Neisseria gonorrhoeae, the prevalence of $T$. vaginalis among older females is especially significant. Napierala et al. $\underline{24}$ summarized clinical laboratory testing of a subacute care population within a high-prevalence STI community using commercially available molecular assays for $\underline{\text { C. trachomatis, }}$ N. gonorrhoeae, and $T$. vaginalis. The $T$. vaginalis detection rate (9.3\%) within a subset of 7,277 females exceeded those of $C$. trachomatis $(5.7 \%)$ and $N$. gonorrhoeae $(1.4 \%$; both $P<0.0002)$. When detection rates were delineated by age decade, $\underline{25}$ the predictable finding of increased $T$. vaginalis detection versus $C$. trachomatis detection in patients aged 31 to 60 years was observed (ig. 2). A T. vaginalis detection rate of greater than $11 \%$ was realized for females over the age of 60 ; however, the small subset did not allow statistical comparison versus $C$. trachomatis detection. Novel findings included elevated $T$. vaginalis detection rates in females under the age of 21 (Fig. 2 ) and significantly increased $T$. vaginalis detection in years 21 to 30 over that of $C$. trachomatis.

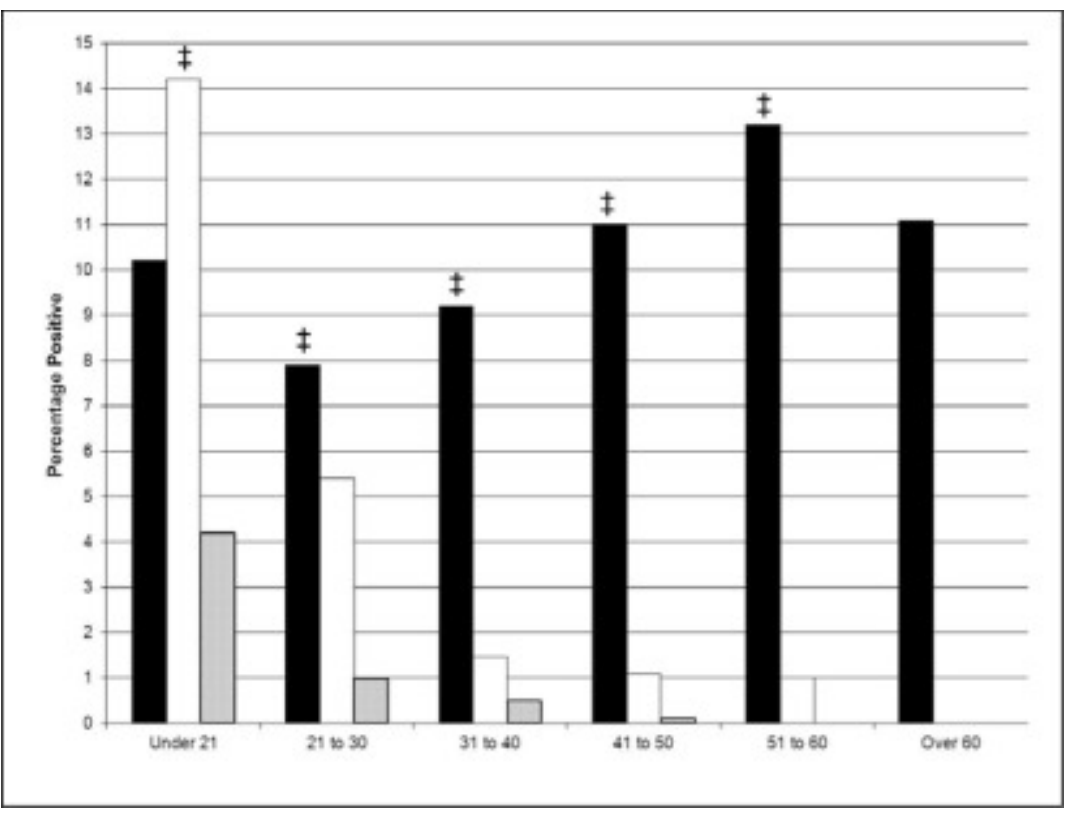

Figure 2. Prevalence of Trichomonas vaginalis (solid bars), Chlamydia trachomatis(open bars), and Neisseria gonorrhoeae (shaded bars) among 7277 females screened by commercial transcription-mediated amplification, delineated by age. $\ddagger$ represents comparisons between $\underline{C}$. trachomatis and $T$. vaginalis detection rates in which $\mathrm{P}$ $<0.05$.

Adapted from data published in, $\underline{25}$ with permission from the publisher. 
Recent investigations have attempted to elucidate the $T$. vaginalis proclivity for older populations. Hearn et al. $\underline{26}$ stratified 264 Baltimore women into age groups of 18 to 44 years and $\geq 45$ years. The prevalence of $T$. vaginalis did not differ between the groups, yet older adults who tested positive for $T$. vaginaliswere more likely to have used marijuana (adjusted odds ratio, $3.1 ; P=0.036$ ) and crack cocaine (adjusted odds ratio, 3.8; $P=0.010$ ) in the previous 6 months. In addition to general immunity decline during the aging process, the authors inferred a link between illicit drug use and increased susceptibility to infection. In vitro T. vaginalis infection models $\underline{27}$ demonstrated suppression of secretory leukocyte protease inhibitor (SLPI), an agent produced by female reproductive

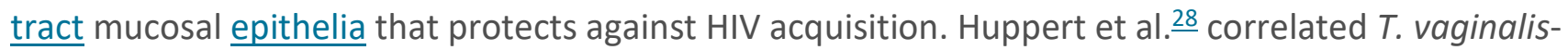
SLPI interaction in a clinical setting, as a $>50 \%$ reduction of this marker was consistent with recurrent $T$. vaginalisinfection. The $T$. vaginalis-SLPI paradigm provides an additional perspective on the necessity for diagnosis of low-level or asymptomatic trichomoniasis. Finally, Fichorova et al. $\underline{29}$ concluded that hormonal contraceptives are associated with alterations in cervical immunity that are dependent upon the presence of genital infections, such as trichomoniasis. In a broad sense, these immune alterations may increase susceptibility to HIV acquisition. The authors posit the potential impact of undiagnosed, asymptomatic genital tract infection on this paradigm.

Past epidemiologic data, often derived from studies utilizing less sensitive diagnostic assays, have suggested that trichomoniasis is largely an urban disease. When considering the dataset in Fig. 2 , it is important to note that $<2 \%$ of those data were gathered from females in acute care. $\underline{25}$ By way of STI phenotype calculation (defined as permutations of $T$. vaginalis, C. trachomatis, and $N$. gonorrhoeae detection from any health care encounter that resulted in detection of at least one STI) and comparison of such data $\underline{25}$ with those from acute care $\underline{30}$ and from a combined subacute and acute care cohort, $\underline{\underline{31}}$ one can ascertain parasite distribution across an entire metropolitan setting. The STI phenotype from subacute care demonstrated the greatest proclivity for sole detection of $T$. vaginalis (54.2\% of health care encounters) (Table 1). In contrast, sole detection of $T$. vaginalis from acute care was $40.7 \%$. Moreover, phenotypes that involved any detection of $T$. vaginalis were $63.5 \%$ and $55.8 \%$ in the subacute care and acute care populations, respectively. The above-mentioned data

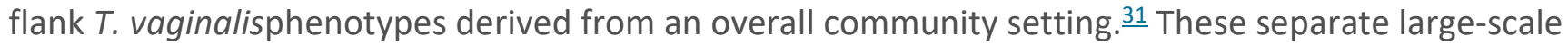
data collections implicate $T$. vaginalis distribution as being widespread throughout a metropolitan setting and signal the necessity for accurate laboratory diagnostics and clinician utilization of those modalities. 
Table 1. High-prevalence community STI phenotypes, determined by commercial transcription-mediated amplification specific for Trichomonas vaginalis, Chlamydia trachomatis, and Neisseria gonorrhoeae within acute care, communitywide, and subacute care samplings of females positive at least one STI

\begin{tabular}{|c|c|c|c|c|c|}
\hline \multicolumn{3}{|c|}{ STI phenotypea } & \multicolumn{3}{|c|}{ Percent patient encounters by specimen source } \\
\hline T. vaginalis & C. trachomatis & N. gonorrhoeae & Acute care $\underline{b}$ & Communitywide $\_$ & Subacute care ${ }^{d}$ \\
\hline+ & - & - & 40.7 & 45.2 & 54.2 \\
\hline+ & + & - & 8.1 & 9.6 & 6.6 \\
\hline+ & - & + & 3.5 & 2.6 & 1.5 \\
\hline+ & + & + & 3.5 & 1.5 & 1.2 \\
\hline- & + & - & 22.2 & 21.0 & 29.1 \\
\hline- & + & + & 4.7 & 5.9 & 2.2 \\
\hline- & - & + & 17.4 & 14.3 & 4.7 \\
\hline
\end{tabular}

${ }^{a}+$, positive TMA screen; -, negative TMA screen.

${ }^{b}$ Eighty-six (33.7\%) encounters had at least one STI. (Data adapted from reference 30with permission of the publisher.)

'Two hundred seventy-three (35.0\%) encounters had at least one STI. (Data adapted from reference 31 with permission of the publisher.)

${ }^{\mathrm{d} O n e}$ thousand thirty-six (14.5\%) encounters had at least one STI. (Data adapted from reference 25 with permission of the publisher.)

Males

Trichomoniasis had been largely uncharacterized in males due to a perceived lack of testing options; however, recent data have further elucidated clinically significant disease in males. Strockij et al. $\underline{32}$ studied prostatic secretions from 203 males with chronic recurrent urethroprostatitis and no detectable bacterial microflora. T. vaginalis was detected in $75.5 \%$ of specimens; amoeboid, spherical, and flagellated forms of the parasite were observed via electron microscopy. Sviben et al. $\underline{33}$ reported a T. vaginalis PCR detection rate of $8.2 \%$ in 500 men with urethritis, while the rate in 200 men without symptoms was $2.2 \%$. T. vaginalis can influence hypogonadism, orchitis, and male infertility. $\underline{34}$

Few data exist regarding the role of T. vaginalis in male HIV acquisition and transmission. Hobbs et al..$\underline{55}$ reported that the median seminal HIV RNA load in Malawi males was increased approximately 2 $\log _{10}$ units in T. vaginalisurethritis. Paz-Bailey et al. $\underline{36}$ investigated 173 HIV-positive males in South Africa with detectable HIV-1 RNA in genital ulcers. Those who were also infected with $T$. vaginalis exhibited increased crude viral loads over those who were not infected (mean difference, $0.62 \log _{10}[95 \%$ confidence interval, 0.07 to $1.2 ; P=0.027])$. The role of the male sexual partner with respect to trichomoniasis and HIV infection should not be overlooked. Chesson et al. $\frac{37}{\text { estimated that }} 750$ instances of new HIV infections in U.S. females were facilitated by concomitant $T$. vaginalis infection; the cost of treating T. vaginalis-attributable HIV infection is \$167 million. 
Over the past 10 years, investigators have attempted to establish an association between prostate carcinoma and antecedent $T$. vaginalisexposure. Studies based on $T$.

vaginalis seroprevalence have demonstrated modest associations with nocturia, large prostate volume, and benign prostatic hyperplasia/lower urinary tract symptom-related outcomes (with prevalence ratios ranging from 1.21 to 1.36 ) $\underline{38}$ and with overall prostate cancer risk (odds ratio, 1.25). $\underline{39}$ Conversely, Mitteregger et al. $\underline{40}$ detected T. vaginalis by PCR in $34 \%$ of tissue specimens with benign prostatic hyperplasia. Stark et al. $\underline{39}$ described significant association between documented $T$. vaginalis infection and lethal prostate cancer (odds ratio, 6.4 [95\% confidence interval, 1.5 to 27.9]) compared to controls with no demonstrable T. vaginalis infection. Akin to HPV triage in cervical carcinoma, studies may be warranted to elucidate a potential role for molecular $T$. vaginalis triage in prostate carcinoma.

Within a high-prevalence STI community, 622 males were screened for C. trachomatis, N. gonorrhoeae, and $T$. vaginalis by RNA amplification; $\underline{41} 6.6 \%$ of the patients yielded a positive result for $T$. vaginalis, with a mean age 39.9 years $(P<0.0001$ versus the mean ages for $C$. trachomatis and $N$. gonorrhoeae). STI agent distribution by age decade (Fig. 3) mimicked data observed in females. The authors also demonstrated no difference in T. vaginalis distribution within majority African American geographic areas of the metropolitan setting (8.9\% detection rate) versus that observed in highly predominantly Caucasian geographic areas of the same metropolitan setting (5.0\% detection rate; $P=0.15)$. These data further show the value of T. vaginalis screening with a broad demographic and geographic basis.

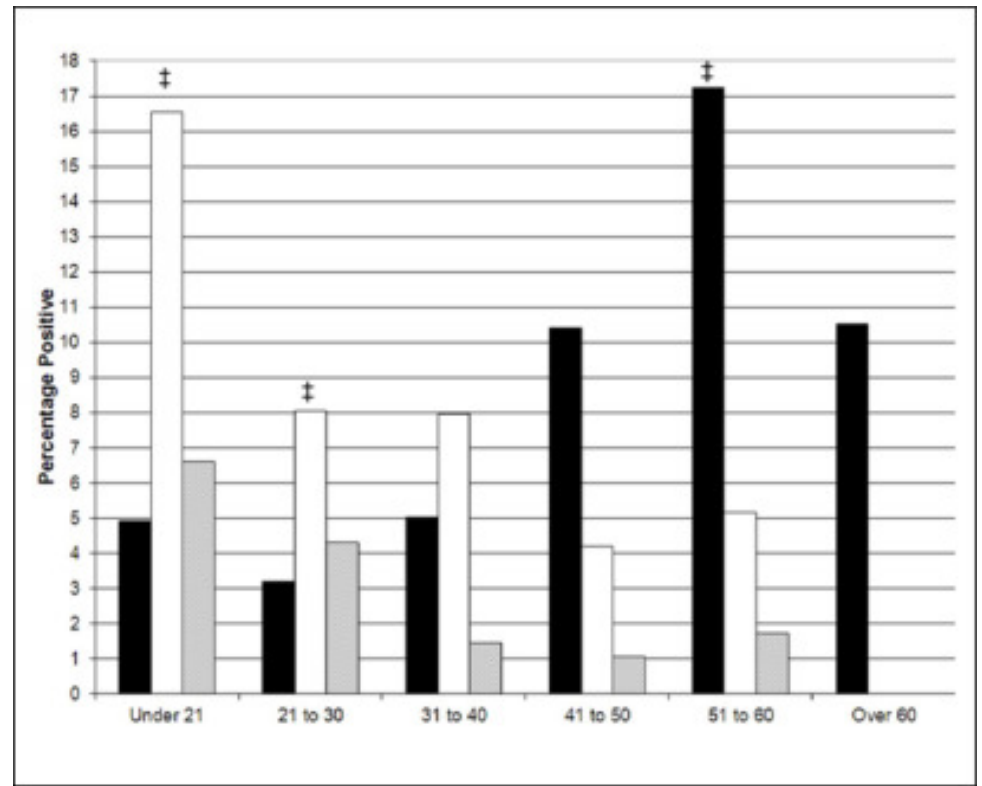

Figure 3. Prevalence of Trichomonas vaginalis (solid bars), Chlamydia trachomatis(open bars), and Neisseria gonorrhoeae (shaded bars) among 622 males screened by commercial transcription-mediated amplification- 
based methods, delineated by age. $¥$ represents comparisons between $T$. vaginalis and $\underline{C}$. trachomatis detection rates in which $\mathrm{P}<0.05$.

Adapted from data published in [41], with permission from the publisher.

\section{Extra-urogenital detection}

Studies have characterized T. vaginalis in non-genitourinary sites. An internet-based STI surveillance program reported a $6.3 \% T$. vaginalis molecular detection rate from self-collected female rectal swabs. $\underline{42}$ Within a subset of these positive patients who also submitted a self-collected vaginal swab, $100 \%$ of the vaginal swab collections were also positive for T. vaginalis (C. Gaydos, personal communication). Munson et al. $\underline{43}$ reported a $2.9 \%$ T. vaginalis pharyngeal detection rate from male STI clinic attendees. Positive results were confirmed by a second molecular assay targeting an alternative $T$. vaginalis sequence; $85.7 \%$ of patients testing positive indicated strictly heterosexual preference, and $38.1 \%$ of $T$. vaginalis-positive pharyngeal specimens were derived from symptomaticpatient encounters.

\section{Laboratory Diagnosis}

\section{Non-molecular modalities}

The success of non-molecular means of $T$. vaginalis detection is largely depenent on the organism burden or disease prevalence. Wet-mount analysis involves collection of a vaginal swab, placement of the swab into physiologic saline (vaginal saline suspension), followed by microscopic observation of the suspension for motile flagellates using a high-power objective. This technique may have its best application in remote and underserved locales that may also experience financial constraints. In a study of Sudanese women attending a gynecologic clinic and experiencing a mean 14.4-day duration of symptoms, Saleh et al. $\underline{3}$ reported $99.2 \%$ wet-mount sensitivity compared to the performance of in-

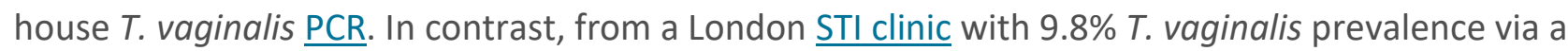
composite reference standard, Nathan et al. [44] reported wet-mount sensitivity of $38 \%$. In a U.S. population with nearly $30 \%$ T. vaginalis prevalence, Nye et al. $\underline{45}$ documented $54.6 \%$ wet-mount sensitivity compared to a composite molecular reference. Decreased sensitivity of wet mount may, in part, be related to the insufficient collection or missed detection of pleomorphic $T$. vaginalis developmental stages. $\underline{46}$

T. vaginalis culture is a past standard for parasite detection, with performance also predicated on the burden of infection or prevalence. Inoculation of vaginal saline suspensions from a population with approximately $85 \%$ T. vaginalis prevalence ${ }^{3}$ into Diamond's medium extrapolated to $100 \%$ analytical sensitivity of culture. In contrast, Nye et al. $\underline{45}$ demonstrated $75 \%$ sensitivity of InPouch TV culture (BioMed Diagnostics, White City, OR) compared to a composite molecular reference. InPouch TV 
evaluation is made by direct microscopy of the culture pouch itself (without aliquoting of culture contents), which may facilitate testing in satellite settings. One advantage inherent to $T$.

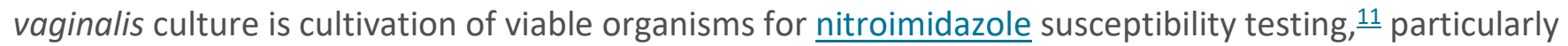
in patients who experience treatment failure.

The performance of a second non-molecular adjunct to microscopy, T. vaginalis antigen detection, is also dependent on geographic prevalence. In the cohort with approximately $85 \% T$.

vaginalis prevalence, $99.6 \%$ sensitivity of the Kalon TV latex agglutination test (Kalon Biological, Surrey, UK) was reported compared to PCR. $\underline{3}$ Abu-Sarkodie et al. $\underline{47}$ documented a kappa index of 0.93 for result agreement between this latex agglutination assay and InPouch TV; molecular diagnostics were not incorporated into the study. Jones et al. $\underline{48}$ assessed the OSOM Trichomonas rapid test (Sekisui Diagnostics, San Diego, CA) using self-collected vaginal swabs from centers in South Africa and Brazil with $T$. vaginalis prevalence of $10 \%$ and $3 \%$, respectively. Compared to a PCR reference, the OSOM Trichomonas rapid test demonstrated $83.3 \%$ and $68.4 \%$ sensitivity, respectively. Nathan et al. $\underline{44}$ documented $92 \%$ sensitivity of the OSOM Trichomonas rapid test at an STI clinic with $9.8 \% T$. vaginalis prevalence. A recent investigation $\underline{49}$ of a U.S. health care system encompassing both low- and high-prevalence STI locales (4.0\% and $21.4 \%$ T. vaginalis detection rates, respectively) documented $35.1 \%$ and $85.7 \%$ sensitivity of the OSOM Trichomonas rapid test in these areas, respectively. Cliniciancollected vaginal swabs submitted for antigen testing were compared to a molecular reference collection. In addition to the potential caveats inherent to antigen-based $T$. vaginalisdetection, it should be noted that FDA clearance has not been granted to these modalities for urine screening or for assessment of male specimens.

\section{Molecular modalities}

The BD Affirm VPIII Microbial Identification Test (Becton Dickinson, Sparks, MD) provides a nucleic acid hybridization format for detection of $T$. vaginalis, in addition to simultaneous assessments for Gardnerella vaginalis and Candida albicans. Recent studies have assessed the $T$. vaginalis component of the assay in the context of a nucleic acid amplification test (NAAT) standard. Within a U.S. population of females with vaginal complaints or history suggestive of STI (5\% $T$. vaginalis prevalence), Andrea and Chapin $\underline{50}$ reported $63.4 \%$ sensitivity of the BD Affirm VPIII Microbial Identification Test compared to a commercial NAAT. The same reference standard was employed by Cartwright et al. $\underline{51}$ in evaluation of a symptomatic 320-female U.S. cohort. The BD Affirm VPIII Microbial Identification Test yielded $46.3 \%$ sensitivity in the population exhibiting $16.9 \%$ T. vaginalis prevalence. Subsequent to analytic assessments of DNA and RNA amplification efficiency, it was postulated that analogous differences exist between commercialized formats of these technologies. $\underline{52}$ This concept was 
validated with an elegant set of in vitro data published by Ikeda-Dantsuji et al. $\underline{53}$ Standardized quantities of $\underline{C}$. trachomatis elementary bodies were distributed into mock clinical specimens; subsequent dilution series demonstrated commercial transcription-mediated amplification (TMA) sensitivity that was 1,000 -fold greater than that of commercial PCR. This difference in sensitivity may also lie in the multiplicity of the target nucleic acid (in particular, rRNA targeted by commercial TMA) and in the removal of endogenous inhibitory agents by an oligonucleotide-based target capture system.

Experimental differences between RNA and DNA amplification have been extrapolated to clinical practice. Schachter et al. $\underline{54}$ demonstrated $44.4 \%$ sensitivity of commercial PCR for detection of $\underline{N}$. gonorrhoeae from rectal specimens compared to commercial TMA. The authors reported just $60.0 \%$ sensitivity of commercial PCR for detection of the agent from pharyngeal swabs. Commercial TMA was also $12.4 \%$ and $14.8 \%$ more sensitive than strand displacement amplification (an alternative form of DNA amplification) in detection of $N$. gonorrhoeae from pharyngeal and rectal sites, respectively. Nye et al. $\underline{45}$ noted a similar paradigm when assessing commercial TMA and an in-house PCR for T. vaginalis. Using a molecularly resolved algorithm, the sensitivities of commercial T. vaginalis TMA on 296 firstvoid female urine, endocervical, and vaginal specimens were $87.5 \%, 89.8 \%$, and $96.6 \%$, respectively. The analogous values derived from PCR were 76.1\%, 80.9\%, and 83.0\%. From 298 first-void male urine and urethral swab specimens, the sensitivity of commercial TMA ranged from $73.8 \%$ (urine) to $95.2 \%$ (urethral swab) when utilizing a molecularly resolved algorithm. The corresponding sensitivity data from PCR were $47.6 \%$ and $54.8 \%$, respectively. The sensitivity of $T$. vaginalis culture from male specimens was $28.6 \%$.

The first FDA-cleared NAAT for T. vaginalis is the TMA-based Aptima Trichomonas vaginalis Assay (Hologic, San Diego, CA), $\underline{55}$ with indications for clinician-collected vaginal swabs, endocervical swabs, and liquid-based cytology collections (PreservCyt solution; Cytyc Corporation, Marlborough, MA). Because the assay can be performed on the same collection tube as $C$. trachomatis/N. gonorrhoeae TMA, a number of laboratories have performed off-label verification of the assay on male first-void urine and urethral swab specimens using laboratory-defined luminescent cutoff values. Napierala et al. $\underline{24}$ reported that the $T$. vaginalis detection rate from males did not differ significantly from $C$. trachomatis detection $(P=0.17)$ and was increased over $N$. gonorrhoeae $(P=0.006)$. The luminescent output from positive specimens did not differ significantly from the values derived from female testing $(P \geq 0.29)$. Increased clinician utilization of a laboratory-verified first-void female urine specimen was also noted during a 3-year testing interval. $\underline{24}$ 
A second FDA-cleared NAAT for T. vaginalis is the strand displacement amplification BD ProbeTec Trichomonas vaginalis Qx Amplified DNA Assay (TV Qx) (BD Diagnostics, Sparks, MD). The indicated specimen sources include female urine, self-collected vaginal swabs, and clinician-collected endocervical swabs. Van Der Pol et al. $\underline{56}$ assessed 838 self-collected vaginal swabs using TV Qx in a multi-center U.S. clinical trial cohort with $12.5 \%$ T. vaginalis prevalence. The kappa value between the TV Qx results and the data from the Aptima Trichomonas vaginalis Assay analysis of clinician-collected swabs from the same participants was $0.938(P=0.09)$. The sensitivity and specificity of TV Qx were 98.3\% and 99.6\%, respectively. Ten specimens yielded Aptima Trichomonas vaginalis Assay-positive/TV Qx-negative results; the converse was true for three specimens.

The AmpliVue Trichomonas Assay (Quidel, San Diego, CA) received FDA clearance in March 2015 for clinician-collected vaginal swabs. Following isothermal helicase-dependent amplification, $T$. vaginalis DNA is detected via lateral-flow colorimetric readout in a self-contained disposable device, with a turnaround time of approximately 1 hour. Unpublished data (C. Gaydos, personal communication) have described the performance of the AmpliVue Trichomonas Assay versus culture, wet mount, and the Aptima Trichomonas vaginalis Assay for 1,132 vaginal swabs. The rapid assay detected $T$. vaginalis from all specimens that were also positive by wet mount or culture analysis. Positive agreement with the Aptima Trichomonas vaginalis Assay data was $88.5 \%$.

The Xpert TV is performed on the GeneXpert system (Cepheid, Sunnyvale, CA). This DNA amplification assay received FDA clearance in October 2015 for female urine, self-collected vaginal swabs, and clinician-collected endocervical swabs. In a proof-of-concept study by Badman et al.,, 50 consecutive urine specimens yielding positive and negative results by an in-house $T$. vaginalis PCR (acquired from 42 males and 72 females) were subjected to automated processing, cell lysis, purification, DNA amplification, and target detection; $97.4 \%$ overall agreement was observed, with 95\% positive agreement.

One commercialized assay for T. vaginalis molecular detection has received Conformité Européene marking. The AmpliSens N. gonorrhoeae/C. trachomatis/M. genitalium/T. vaginalis-Multiprime-FRT PCR kit (InterLabScience, Moscow, Russia) is a multiplex real-time PCR assay that requires an off-line DNA extraction step. Rumyantseva et al. $\underline{58}$ investigated its performance using first-void urine from 554 males, first-void urine from 498 females, and 209 vaginal swabs. T. vaginalis assessment was difficult on the basis of just one positive reference specimen in the evaluation. The assay was also limited in its capacity to detect Mycoplasma genitalium from both male and female specimens.

Additional modalities under development include a point-of-care cartridge (Atlas Genetics, Wiltshire, UK) that manages DNA extraction, amplification, and detection within 30 minutes using molded 
subcircuits. The final output is an electrochemical signal obtained using differential pulse voltammetry. Pearce et al. $\underline{59}$ assessed a single-analyte $T$. vaginalis assay with 90 self-collected vaginal swabs previously tested by the Aptima Trichomonas vaginalis Assay. Sensitivity and specificity indices of 95.5\% and $95.7 \%$, respectively, were reported. The detection limit of the assay was five $T$. vaginalis organisms. As part of an investigational FilmArray nested-PCR panel for the identification of nine STI agents (BioFire Diagnostics, Salt Lake City, UT), Kriesel et al. $\underline{60}$ identified T. vaginalis DNA from 9 of 295 clinical specimens. However, not all the specimens were assessed with a predicate device. Finally, recent proof-of-concept studies have discussed detection of $T$. vaginalis by loop-mediated $\underline{\text { isothermal amplification }}{ }^{61}$ and PCR-based microarray.

\section{Value of $T$. vaginalis screening}

The CDC recommendations for T. vaginalis diagnostic testing, using highly sensitive and specific modalities, apply to females seeking care for vaginal discharge. $\frac{11}{1}$ The screening recommendations extend to persons receiving care in high-prevalence settings (correctional facilities and STI clinics) and to individuals with high-risk STI behavior (commercial sex workers, illicit drug use, and persons with multiple sexual partners or a history of STI). Moreover, local T. vaginalis epidemiology may influence screening decisions. $\underline{\underline{11}}$

The scope of trichomoniasis may extend beyond these demographics, as Tomas et al. $\underline{63}$ described scenarios in acute care in which STI (including $T$. vaginalis infection) was underdiagnosed in females in favor of empiric treatment for urinary tract infection. Retrospective chart reviews of 234 symptomatic females receiving $C$. trachomatis and $N$. gonorrhoeae screening $\underline{64}$ revealed that only 31 were screened for $T$. vaginalis. While a laboratory diagnosis of $T$. vaginalis was rendered in just $1.3 \%(3 / 234)$ of eligible females, 17 patients were administered anti-trichomonad therapy ( $82.3 \%$ without significant laboratory data). Following commencement of an algorithm that automatically added $T$. vaginalis laboratory assessment to those for $C$. trachomatis and $N$. gonorrhoeae, $T$. vaginalis was detected in 18.3\% (39/213) of eligible females; $95.8 \%$ of $T$. vaginalis laboratory diagnoses resulted in therapeutic regimens, while only $25 \%$ of overall anti-trichomonad regimens were administered on an empiric basis. Data from a high-prevalence STI community $\underline{65}$ revealed that introduction of $T$. vaginalis TMA to the testing options resulted in a significant increase in laboratory assessments for $T$. vaginalis compared to an interval when only wet-mount testing was offered. In addition, significant reductions in both clinician requisition of wet-mount analysis and clinician performance of point-ofcare wet-mount microscopy was observed. Although NAAT yielded 38\% more $T$. vaginalis detection than culture in one study, the prospect of universal screening may be somewhat tempered in lowerprevalence settings. $?$ 


\section{Asymptomatic patients}

One concern about $T$. vaginalis molecular screening is the unnecessary laboratory diagnosis of asymptomatic patients, $\frac{66}{6}$ with a corollary being that less sensitive assays that detect higher organism burdens are more clinically relevant. However, Muzny et al. $\underline{\underline{2}}$ demonstrated that $58.5 \%$ of 301 females with wet-mount-negative/TMA-positive results reported urogenital symptoms of vaginal discharge, vaginal odor, and/or dysuria. This datum was similar to the $65.7 \%$ symptomatic rate of 720 females with wet-mount-positive/TMA-positive results (adjusted odds ratio, 1.00; $P=0.98$ ). Munson et al.99 reported that $56.8 \%$ of antigen assay-negative/TMA-positive patients from a low-prevalence STI community had symptoms of urogenital infection, while $75.0 \%$ of patients from the same setting with antigen assay-positive/TMA-positive results were symptomatic $(P=0.17)$. In addition, symptomatic status did not impact concordant and discordant antigen assay/TMA frequencies in a higherprevalence STI population $(P=0.54)$. These data support the hypothesis that the performance of nonmolecular $T$. vaginalis assays is more accurately predicated on disease prevalence than on symptomatic status.

Detection of asymptomatic trichomoniasis is also important, because studies $\underline{67,68}$ have documented persistent indolent $T$. vaginalis infection based on laboratory detection of the organism following previously negative test results in the face of sexual abstinence. Munson et al. .99 stated that $27 \%$ of patients in a low-prevalence STI setting with antigen assay-negative/(retrospective) TMA-positive results returned for clinical STI evaluation.

Two papers discuss this paradigm in the context of prevalent/incident $T$. vaginalis infection. A ninecenter study $\underline{\underline{69}}$ characterized the initial T. vaginalis prevalence as $14.6 \%$. The cumulative 6 -month incidence of $T$. vaginalis infection (defined as $T$. vaginalis detection in patients with either a negative $T$. vaginalis result at baseline or a positive result at baseline and receiving anti-trichomonad therapy) was 7.5\%. Patients more likely to have incident $T$. vaginalis infections included those with baseline HIV infection (relative risk, 1.59) and baseline T. vaginalis infection (relative risk, 3.37). Because nearly $50 \%$ of patients positive for $T$. vaginalis at baseline received therapy, the authors speculate that incident $T$. vaginalis was related to re-infection or treatment failure. Gaydos et al..$^{70}$ studied 304 female repeat participants in an internet STI screening program. Within this cohort, the $T$. vaginalis TMA detection rate was $7.9 \%$ during the first assessment, with $9.2 \%$ detection upon repeat testing (60\% reporting symptoms). T. vaginalisdetection among repeat participants was also associated with previous detection of $T$. vaginalis $(P<0.05)$. 


\section{Final notes on specimen collection}

Recent data from the CDC포 support the collection of vaginal swabs for laboratory detection of $C$. trachomatis and $N$. gonorrhoeae. Such collections are preferred for assessment of $T$.

vaginalis infection; this preference is substantiated by data from Nye et al. $\underline{45}$ Other studies in the literature espouse first-void urine as a viable specimen option for detection of $T$. vaginalis in females. Napierala et al. $\underline{24}$ reported a $12.6 \% \mathrm{~T}$. vaginalisdetection rate from first-void urine in contrast to $8.9 \%$ and $8.6 \%$ rates from endocervical and vaginal specimens, respectively. A follow-up assessment of 2,478 females from a large metropolitan cohort (including both high- and low-prevalence locales) ${ }^{72}$ reported increased $T$. vaginalis detection from first-void urine (11.3\%) compared to endocervical specimens (8.2\%; $P=0.04)$ and a trend toward increased detection from first-void urine versus a combined endocervical/vaginal specimen data set $(P=0.06)$. The above-mentioned studies are limited because data analysis was based on the specimens collected by clinicians during routine medical practice; multiple specimens were not collected from the same patient. However, Andrea and Chapin $\underline{50}$ reported $11.5 \%$ and $3.2 \%$ T. vaginalis detection rates from female first-void urine and endocervical collection, respectively $(P<0.0002)$.

When $C$. trachomatis and T. vaginalis detection rates were compared in a demographic (under age 21) in which $T$. vaginalis is not typically considered within an STI differential diagnosis (Fig. 2 ), the $C$. trachomatis detection rate among approximately 1,500 females was higher than that of $T$. vaginalis $(P=0.001)$. However, when the detection rates were stratified by specimen source, $\underline{25}$ the $T$. vaginalis detection rate (11.2\%) among nearly 300 females who were screened with first-void urine rivaled the rate for $C$. trachomatis $(10.9 \% ; P=0.92)$. First-void urine screening seemingly eliminated a disparity between African American and Caucasian female demographics with respect to $T$. vaginalis detection. Overall, TMA detection of the parasite in African American majority geographic locales across a large metropolitan area was $11.7 \%$ compared to $9.0 \%$ in predominantly Caucasian locales $(P=0.004)$. When stratified by first-void urine collection, $T$. vaginalis detection rates exhibited minimal difference (14.4\% and $12.5 \%$ in African American and Caucasian majority locales, respectively; $P=0.54) . \underline{25}$

Gaydos et al. $\underline{73}$ described 3.7\% detection of T. vaginalis RNA from 1,699 males using self-collected penile-meatal swabs in the context of an Internet screening program. Similar to previous studies of trichomoniasis epidemiology in males, the associated risk factors included age 30 to 39 years (odds ratio, 6.63) and age $>40$ years (odds ratio, 5.31). In a second cohort, Dize et al. $\underline{74}$ investigated concomitant submission of self-collected penile-meatal swabs and first-void urine from 634 males. From 56 patients with detectable $T$. vaginalis infection via TMA, the swab sensitivity was $80.4 \%$ and the 
first-void urine sensitivity was 39.3\%; similar disparities in sensitivity were observed for $C$. trachomatis and N. gonorrhoeae.

\section{Conclusion}

Evaluation of reports regarding past modalities for diagnosis of trichomoniasisis compromised by an evolving molecular reference standard. This is further confounded by analytical sensitivity differences between DNA and RNA amplification methods demonstrated both in vitro and in the clinical setting. However, these recently commercialized, highly accurate diagnostic modalities, particularly those based on TMA, are facilitating "new" outlooks on the "old" epidemiology of trichomoniasis. This clinical entity is now widely distributed in a geographic setting, in both genders, and in wide range of age demographics. Evidence increasingly supports the value of $T$. vaginalismolecular diagnostics in the assessment of both symptomatic and asymptomatic populations. In conclusion, molecular assays for trichomoniasis, including those adapted to laboratory-modified testing formats, have the potential to impact public health and clinical practice.

\section{References}

${ }^{1}$ CL Satterwhite, E Torrone, E Meites, EF Dunne, R Mahajan, MC Ocfemia, et al. Sexually transmitted infections among US women and men: prevalence and incidence estimates, 2008. Sex Transm Dis, 40 (2013), pp. 187-193

CA Muzny, RJ Blackburn, EL Sinsky, Austin, JR Schwebke. Added benefit of nucleic acid amplification testing for the diagnosis of Trichomonas vaginalis among men and women attending a sexually transmitted diseases clinic. Clin Infect Dis, 59 (2014), pp. 834-841

${ }^{3}$ AM Saleh, HS Abdalla, AB Satti, SM Babiker, GI Gasin, I Adam. Diagnosis of Trichomonous (sic) vaginalis by microscopy, latex agglutination, diamond's media, and PCR in symptomatic women, Khartoum, Sudan. Diagn Pathol, 9 (2014), p. 49

${ }^{4} \mathrm{R}$ Guy, J Ward, H Wand, A Rumbold, L Garton, B Hengel, et al. Coinfection with Chlamydia trachomatis, Neisseria gonorrhoeae, and Trichomonas vaginalis: a cross-sectional analysis of positivity and risk factors in remote Australian Aboriginal communities. Sex Transm Infect, 91 (2015), pp. 201-206

${ }^{5}$ RA Wangnapi, S Soso, HW Unger, C Sawera, M Ome, AJ Umbers, et al. Prevalence and risk factors for Chlamydia trachomatis, Neisseria gonorrhoeaeand Trichomonas vaginalis infection in pregnant women in Papua New Guinea. Sex Transm Infect, 91 (2015), pp. 194-200

${ }^{6} \mathrm{~N}$ Khatib, C Bradbury, V Chalker, GCKW Koh, E Smit, S Wilson, et al. Prevalence of Trichomonas vaginalis, Mycoplasma genitalium and Ureaplasma urealyticum in men with urethritis attending an urban sexual health clinic. Int J STD AIDS, 26 (2015), pp. 388-392

${ }^{7} E$ Hathorn, A Ng, M Page, J Hodson, C Gaydos, JDC Ross. A service evaluation of the Gen-Probe nucleic acid amplification test for Trichomonas vaginalis: should it change whom we screen for infection? Sex Transm Infect, 91 (2015), pp. 81-86 
${ }^{8}$ GGG Donders, CE Depuydt, J-P Bogers, AJ Vereecken. Association of Trichomonas vaginalis and cytological abnormalities of the cervix in low risk women. PLoS One, 8 (2013), p. e86266

${ }^{9}$ Kim HJ, Park JK, Park SC, Kim YG, Choi H, Ko Jl et al. The prevalence of causative organisms of community-acquired urethritis in an age group at high risk for sexually transmitted infections in Korean soldiers. J R Army Med Corps doi:10.1136/jramc-2015-000488.

$10 \mathrm{JM}$ Bygott, JM Robson. The rarity of Trichomonas vaginalis in urban Australia. Sex Transm Infect, 89 (2013), pp. 509-513

${ }^{11}$ Centers for Disease Control and Prevention. Sexually transmitted diseases treatment guidelines. MMWR Recomm Rep, 64 (RR-03) (2015), pp. 1-137

$12 \mathrm{P}$ Heine, JA McGregor. Trichomonas vaginalis: a reemerging pathogen. Clin Obstet Gynecol, 36 (1993), pp. 137-144

$\underline{13}$ FJ Bowden, GP Garnett. Trichomonas vaginalis epidemiology: parameterising and analyzing a model of treatment interventions. Sex Transm Infect, 76 (2000), pp. 248-256

${ }^{14}$ E Meites, CA Gaydos, MM Hobbs, P Kissinger, P Nyirjesy, JR Schwebke, et al. A review of evidencebased care of symptomatic trichomoniasis and asymptomatic Trichomonas vaginalis infections. Clin Infect Dis, 61 (Suppl 8) (2015), pp. S837-S848

15JR Mann, S McDermott, T Gill. Sexually transmitted infection is associated with increased risk of preterm birth in South Carolina women insured by Medicaid. J Matern Fetal Neonatal Med, 23 (2010), pp. 563-568

${ }^{16} \mathrm{ML}$ Shew, JD Fortenberry, W Tu, BE Juliar, BE Batteiger, B Quadadri, et al. Association of condom use, sexual behaviors, and sexually-transmitted infections with the duration of genital human papillomavirus infection among adolescent women. Arch Pediatr Adolesc Med, 160 (2006), pp. 151-156

17J Ting, N Mugo, J Kwatampora, C Hill, M Chitwa, S Patel, et al. High-risk human papillomavirus messenger RNA testing in physician- and self-collected specimens for cervical lesion detection in high-risk women, Kenya. Sex Transm Dis, 40 (2013), pp. 584-589

18TL Mullins, BJ Rudy, CM Wilson, H Sucharew, JA Kahn. Incidence of sexually-transmitted infections in HIV-infected and HIV-uninfected adolescents in the USA. Int J STD AIDS, 24 (2013), pp. 123-127

${ }_{19}^{19}$ N Mavedzenge, BV Pol, H Cheng, ET Montgomery, K Blanchard, G de Bruyn, et al. Epidemiology synergy of Trichomonas vaginalis and HIV in Zimbabwean and South African women. Sex Transm Dis, 37 (2010), pp. 460-466

20.JP Hughes, JM Baeten, JR Lingappa, AS Magaret, A Wald, G de Bruyn, et al. Determinants of percoital-act HIV-1 infectivity among African HIV-1-serodiscordant couples. J Infect Dis, 205 (2012), pp. 358-365

21PC Guenther, WE Secor, CS Dezzutti. Trichomonas vaginalis-induced epithelial monolayer disruption and human immunodeficiency virus type 1 (HIV-1) replication: implications for the sexual transmission of HIV-1. Infect Immun, 73 (2005), pp. 4155-4160

22P Kissinger, A Amedee, RA Clark, J Dumestre, KP Theall, L Myers, et al. Trichomonas vaginalis treatment reduces vaginal HIV-1 shedding. Sex Transm Dis, 36 (2009), pp. 11-16 
${ }^{23}$ EB Quinlivan, SN Patel, CA Grodensky, CE Golin, HC Tien, MM Hobbs. Modeling the impact of Trichomonas vaginalis infection on HIV transmission in HIV-infected individuals in medical care. Sex Transm Dis, 39 (2012), pp. 671-677

${ }^{24} \mathrm{M}$ Napierala, E Munson, KL Munson, T Kramme, C Miller, J Burtch, et al. Three-year history of transcription-mediated amplification-based Trichomonas vaginalis analyte-specific reagent testing in a subacute care patient population. J Clin Microbiol, 49 (2011), pp. 4190-4194

${ }^{25} \mathrm{E}$ Munson, T Kramme, M Napierala, KL Munson, C Miller, JE Hryciuk. Female epidemiology of transcription-mediated amplification-based Trichomonas vaginalis detection in a metropolitan setting with a high prevalence of sexually transmitted infection. J Clin Microbiol, 50 (2012), pp. 3927-3931

${ }^{26}$ LE Hearn, NE Whitehead, EM Dunne, WW Latimer. Correlates of Trichomonas vaginalis among middle age and older adults who use drugs. Subst Use Misuse, 50 (2015), pp. 1501-1509

27RN Fichorova, OR Buck, HS Yamamoto, T Fashemi, HY Dawood, B Feshemi, et al. The villain team-up or how Trichomonas vaginalis and bacterial vaginosis alter innate immunity in concert. Sex Transm Infect, 89 (2013), pp. 460-466

28fS Huppert, B Huang, C Chen, HY Dawood, RN Fichorova. Clinical evidence for the role of Trichomonas vaginalis in regulation of secretory leukocyte protease inhibitor in the female genital tract. J Infect Dis, 207 (2013), pp. 1462-1470

${ }^{29}$ RN Fichorova, P-L Chen, CS Morrison, GF Doncel, K Mendonca, C Kwok, et al. The contribution of cervicovaginal infections to the immunomodulatory effects of hormonal contraception. MBio, 6 (2015), p. e00221. e00215

${ }^{30}$ E Munson, M Napierala, J Basile, C Miller, J Burtch, JE Hryciuk, et al. Trichomonas vaginalis transcription-mediated amplification-based analyte-specific reagent and alternative target testing of primary clinical vaginal saline suspensions. Diagn Microbiol Infect Dis, 68 (2010), pp. 66-72

31E Munson, M Napierala, R Olson, T Endes, T Block, JE Hryciuk, et al. Impact of Trichomonas vaginalis transcription-mediated amplification-based analyte-specific-reagent testing in a metropolitan setting of high sexually-transmitted disease prevalence. J Clin Microbiol, 46 (2008), pp. 3368-3374

$\underline{32}$ AV Strockij, AA Gavrusev, LV Rubanik, NN Poleshchuk. Is a nonbacterial prostatis nonbacterial? (Russian) Urologiia, 4 (2015), pp. 102-106

${ }^{33}$ M Sviben, EM Missoni, T Meštrović, G Vojnović, GM Galinović. Epidemiology and laboratory characteristics of Trichomonas vaginalis infection in Croatian men with and without urethritis syndrome: a case-control study. Sex Transm Infect, 91 (2015), pp. 360-364

${ }^{34} \mathrm{G}$ Lloyd, JR Case, D De Frias, RE Brannigan. Trichomonas vaginalis orchitis with associated severe oligoasthenoteratospermia and hypogonadism. J Urol, 170 (2003), p. 924

${ }^{35} \mathrm{MM}$ Hobbs, P Kazembe, AW Reed, WC Miller, E Nkata, D Zimba, et al. Trichomonas vaginalis as a cause of urethritis in Malawian men. Sex Transm Dis, 26 (1999), pp. 381-387 
${ }^{36} \mathrm{G}$ Paz-Bailey, M Sternberg, AJ Puren, L Steele, DA Lewi. sDeterminants of HIV type 1 shedding from genital ulcers among men in South Africa. Clin Infect Dis, 50 (2010), pp. 1060-1067

${ }^{37} \mathrm{HW}$ Chesson, JM Blandford, SD Pinkerton. Estimates of the annual number and cost of new HIV infections among women attributable to trichomoniasis in the United States. Sex Transm Dis, 31 (2004), pp. 547-551

38 BN Breyer, W-Y Huang, CS Rabkin, JF Alderete, R Pakpahan, et al. Sexually transmitted infections, benign prostatic hyperplasia and lower urinary tract symptom-related outcomes: results from the Prostate, Lung, Colorectal and Ovarian Cancer Screening Trial. BJU Int, 117 (2016), pp. 145154

39JR Stark, G Judson, JF Alderete, V Mundodi, AS Kucknoor, EL Giovannucci. Prospective study of Trichomonas vaginalis infection and prostate cancer incidence and mortality: Physicians' Health Study. J Natl Cancer Inst, 101 (2009), pp. 1406-1411

40D Mitteregger, SW Aberle, A Makristathis, J Walochnik, W Brozek, M Marberger, et al. High detection rate of Trichomonas vaginalis in benign hyperplastic prostatic tissue. Med Microbiol Immunol, 201 (2012), pp. 113-116

${ }^{41} \mathrm{KL}$ Munson, M Napierala, E Munson, RF Schell, T Kramme, C Miller, et al. Screening of male patients for Trichomonas vaginalis with transcription-mediated amplification in a community with a high prevalence of sexually transmitted infection. J Clin Microbiol, 51 (2013), pp. 101-104

42J Ladd, Y-H Hsieh, M Barnes, N Quinn, M Jett-Goheen, CA Gaydos. Female users of internet-based screening for rectal STIs: descriptive statistics and correlates of positivity. Sex Transm Infect, 90 (2014), pp. 485-490

${ }^{43}$ E Munson, D Wenten, P Phipps, R Gremminger, MK Schuknecht, M Napierala, et al. Retrospective assessment of transcription-mediated amplification-based screening for Trichomonas vaginalis in male sexually-transmitted infection clinic patients. J Clin Microbiol, 51 (2013), pp. 1855-1860

${ }_{44}$ B Nathan, J Appiah, P Saunders, D Heron, T Nichols, R Brum, et al. Microscopy outperformed in a comparison of five methods for detecting $T$. vaginalis in symptomatic women. Int J STD AIDS, 26 (2015), pp. 251-256

${ }_{45} \mathrm{MB}$ Nye, JR Schwebke, BA Body. Comparison of APTIMA Trichomonas vaginalis transcriptionmediated amplification to wet mount microscopy, culture, and polymerase chain reaction for diagnosis of trichomoniasis in men and women. Am J Obstet Gynecol, 200 (2009), pp. 188.e1188.e7

${ }^{46} \mathrm{R}$ Arroyo, A González-Robles, A Martínez-Palomo, JF Alderete. Signalling of Trichomonas vaginalis for amoeboid transformation and adhesion synthesis follows cytoadherence. $\mathrm{Mol}$ Microbiol, 7 (1993), pp. 299-309

${ }^{47} Y$ Abu-Sarkodie, BK Opoku, KA Danso, HA Weiss, D Mabey. Comparison of latex agglutination, wet preparation, and culture for the detection of Trichomonas vaginalis. Sex Transm Infect, 80 (2004), pp. 201-203 
${ }^{48}$ HE Jones, SA Lippman, Caiaffa-Filho, T Young, JHHM van de Wijgert. Performance of a rapid self-test for detection of Trichomonas vaginalis in South Africa and Brazil. J Clin Microbiol, 51 (2013), pp. 1037-1039

${ }^{49} \mathrm{KL}$ Munson, Napierala, E Munson. Suboptimal Trichomonas vaginalis antigen test performance in a low-prevalence sexually transmitted infection community. J Clin Microbiol, 54 (2016), pp. 500501

${ }^{50}$ SB Andrea, KC Chapin. Comparison of Aptima Trichomonas vaginalis transcription-mediated amplification assay and BD Affirm VPIII for detection of Trichomonas vaginalis in symptomatic women: performance parameters and epidemiological implications. $J$ Clin Microbiol, 49 (2011), pp. 866-869

${ }^{51}$ CP Cartwright, BD Lembke, K Ramachandran, BA Body, MB Nye, CA Rivers. Comparison of nucleic acid amplification assays with BD Affirm VPIII for diagnosis of vaginitis in symptomatic women. J Clin Microbiol, 51 (2013), pp. 3694-3699

52 J Schachter, EW Hook, DH Martin, D Willis, P Fine, D Fuller, et al. Confirming positive results of nucleic acid amplification tests (NAATs) for Chlamydia trachomatis: all NAATs are not created equal. J Clin Microbiol, 43 (2005), pp. 1372-1373

53 Y Ikeda-Dantsuji, I Konomi, A Nagayama. In vitro assessment of the APTIMA Combo 2 assay for the detection of Chlamydia trachomatis using highly purified elementary bodies. J Med Microbiol, 54 (2005), pp. 357-360

54J Schachter, J Moncada, S Liska, C Shayevich, JD Klausner. Nucleic acid amplification tests in the diagnosis of chlamydial and gonococcal infections of the oropharynx and rectum in men who have sex with men. Sex Transm Dis, 35 (2008), pp. 637-642

55JR Schwebke, MM Hobbs, SN Taylor, AC Sena, MG Catania, BS Weinbaum, et al. Molecular testing for Trichomonas vaginalis in women: results from a prospective U.S. clinical trial. J Clin Microbiol, 49 (2011), pp. 4106-4111

56 B Van Der Pol, JA Williams, SN Taylor, CL Cammarata, CA Rivers, BA Body, et al. Detection of Trichomonas vaginalis DNA by use of self-obtained vaginal swabs with the BD ProbeTec $\mathbf{Q}^{\mathbf{x}}$ assay on the BD Viper system. J Clin Microbiol, 52 (2014), pp. 885-889

${ }^{57}$ SG Badman, LM Causer, R Guy, SN Tabrizi, F Francis, B Donovan, et al. A preliminary evaluation of a new GeneXpert (GX) molecular point-of-care test for the detection of Trichomonas vaginalis. Sex Transm Infect, 92 (2016), pp. 350-352

58 T Rumyantseva, D Golparian, CS Nilsson, E Johansson, M Falk, H Fredlund, et al. Evaluation of the new AmpliSens multiplex real-time PCR assay for simultaneous detection of Neisseria gonorrhoeae, Chlamydia trachomatis, Mycoplasma genitalium, and Trichomonas vaginalis. APMIS, 123 (2015), pp. 879-886

${ }_{59}^{5} D M$ Pearce, DN Styles, JP Hardick, CA Gaydos. A new rapid molecular point-of-care assay for Trichomonas vaginalis: preliminary performance data. Sex Transm Infect, 89 (2013), pp. 495-497 
${ }^{60}$ Kriesel JD, Bhatia AS, Barrus C, Vaughn M, Gardner J, Crisp RJ. Multiplex PCR testing for nine different sexually transmitted diseases. Int J STD AIDS doi:10.1177/0956462415615775.

61JCB Reyes, JAA Solon, WL Rivera. Development of a loop-mediated isothermal amplification assay for detection of Trichomonas vaginalis. Diagn Microbiol Infect Dis, 79 (2014), pp. 337-341

62OJ Kweon, J-H Choi, UH Song, AJ Park. Performance evaluation of a DNA chip assay in the identification of major genitourinary pathogens. J Microbiol Methods, 109 (2015), pp. 117-122

${ }_{63}^{6} \mathrm{ME}$ Tomas, D Getman, CJ Donskey, MT Hecker. Overdiagnosis of urinary tract infection and underdiagnosis of sexually transmitted infection in adult women presenting to an emergency department. J Clin Microbiol, 53 (2015), pp. 2686-2692

64 HM Territo, BH Wrotniak, S Bouton, GR Burstein. A new strategy for Trichomonas testing female adolescents in the emergency department. J Pediatr Adolesc Gynecol, 29 (2016), pp. 378-381

65E Munson, C Miller, M Napierala, T Kramme, R Olson, KL Munson, et al. Assessment of screening practices in a subacute clinical setting following introduction of Trichomonas vaginalis nucleic acid amplification testing. Wisc Med J, 111 (2012), pp. 233-236

66 LV Smith, F Sorvillo, T Kuo. Implications of Trichomonas vaginalis nucleic acid amplification testing on medical training and practice. J Clin Microbiol, 51 (2013), p. 1650

67TA Peterman, LH Tian, CA Metcalf, CK Malotte, SM Paul, JM Douglas Jr. Persistent, undetected Trichomonas vaginalis infection? Clin Infect Dis, 48 (2009), pp. 259-260

$68 \mathrm{M}$ Gatski, P Kissinger. Observation of probable persistent, undetected Trichomonas vaginalis infection among HIV-positive women. Clin Infect Dis, 51 (2010), pp. 114-115

${ }_{69} \mathrm{ML}$ Alcaide, DJ Feaster, R Duan, S Cohen, C Diaz, JG Castro, et al. The incidence of Trichomonas vaginalis infection in women attending nine sexually transmitted diseases clinics in the USA. Sex Transm Infect, 92 (2016), pp. 58-62

${ }^{70}$ CA Gaydos, M Barnes, M Jett-Goheen, N Quinn, P Whittle, T Hogan, et al. Characteristics and predictors of women who obtain rescreening for sexually-transmitted infections using the www.iwantthekit.org screening program. Int J STD AIDS, 24 (2013), pp. 736-744

71J Papp, J Schachter, C Gaydos, B Van Der Pol. Recommendations for the laboratory-based detection of Chlamydia trachomatisand Neisseria gonorrhoeae-2014. MMWR Recomm Rep, 64 (RR02) (2014), pp. 1-24

72E Munson, H Bykowski, KL Munson, M Napierala, PJ Reiss, RF Schell, et al. Clinical laboratory assessment of Mycoplasma genitalium transcription-mediated amplification using primary female urogenital specimens. J Clin Microbiol, 54 (2016), pp. 432-438

${ }^{73}$ CA Gaydos, MR Barnes, N Quinn, M Jett-Goheen, YH Hsieh. Trichomonas vaginalis infection in men who submit self-collected penile swabs after internet recruitment. Sex Transm Infect, 89 (2013), pp. 504-508

74L Dize, P Agreda, N Quinn, MR Barnes, Y-H Hsieh, CA Gaydos. Comparison of self-obtained penilemeatal swabs to urine for the detection of $C$. trachomatis, $N$. gonorrhoeae and $T$. vaginalis. Sex Transm Infect, 89 (2013), pp. 305-307 
Uncited Reference

${ }^{75} \mathrm{C}$ Gaydos, J Hardick. Point of care diagnostics for sexually transmitted infections: perspectives and advances. Expert Rev Anti Infect Ther, 12 (2014), pp. 657-672 